\title{
LOCALISATION OF CHROMOSOME BREAKAGE AT MEIOSIS
}

\author{
H. REES and J. B. THOMPSON
}

Agricultural Research Council's Unit of Biometrical Genetics, Department of Genetics, University of Birmingham

Received 5.i.55

\section{INTRODUCTION AND HISTORY OF THE MATERIAL}

THE dependence of induced chromosome structural change on the genotype is clear wherever, as has often been observed (see Koller, 1954), different individuals show different frequencies of change after subjection to the same treatment. Indeed it is sometimes possible to go further and relate the occurrence of spontaneous structural change-breakage, reunion and so on-to unbalance of the genotype resulting from wide hybridisation (as in Bromus; Walters, I95I) or forced inbreeding (as in rye ; Lamm, I936; Müntzing and Akdik 1948). The present report deals with a new example of structural change found at meiosis in a strain of inbred rye. Its consequences are sufficiently few and simple to make a detailed analysis possible, and the abnormality has a further and special interest in the differentiation it reveals along the chromosomes.

The inbred line, since the $23^{\text {rd }}$ generation, has been continued as two sub-lines, $\mathrm{P}_{13}$ and $\mathrm{P}_{14}$. When these sub-lines were first examined, in the 27 th generation, they were found to differ in chromosome behaviour at meiosis (Rees, 1955). In plants of $\mathrm{P}_{13}$ there was much fragmentation and bridge formation at first anaphase, whereas plants of $\mathrm{P}_{14}$ had little. There was also, in conjunction with the breakage in $\mathrm{P}_{1} 3$ a lower terminalisation of chiasmata at first metaphase. These differences must be attributed either to mutation or to residual heterozygosity of the parent line.

The following is an account of meiosis in $\mathrm{P}_{1} 3$.

\section{BREAKAGE AND INTERPRETATION}

At first metaphase less than 10 per cent. of the total breakage is detectable. Free chromosome fragments lie off the metaphase plate in about I per cent. of p.m.c. (fig. $\mathrm{I} b$ ), and in about another 5 per cent. bivalents are deformed by breakage and reunion superimposed upon chiasma formation (figs. $1 a-f$, plate figs. I and 2).

Breaks in metaphase bivalents are of the $\mathrm{B}^{\prime \prime}$ (chromosome) type, since each break involves both sister strands of a chromosome. The rarity of acentric fragments at metaphase suggests that the breakage takes place after pairing, i.e. during pachytene before splitting.

The manifestation of a break at metaphase depends on the broken 
ends being forced apart by the normal repulsions acting on an abnormal structure (fig. 2), and this happens when :-

(i) the break is between the centromere and a chiasma, or

(ii) the break is between two chiasmata.

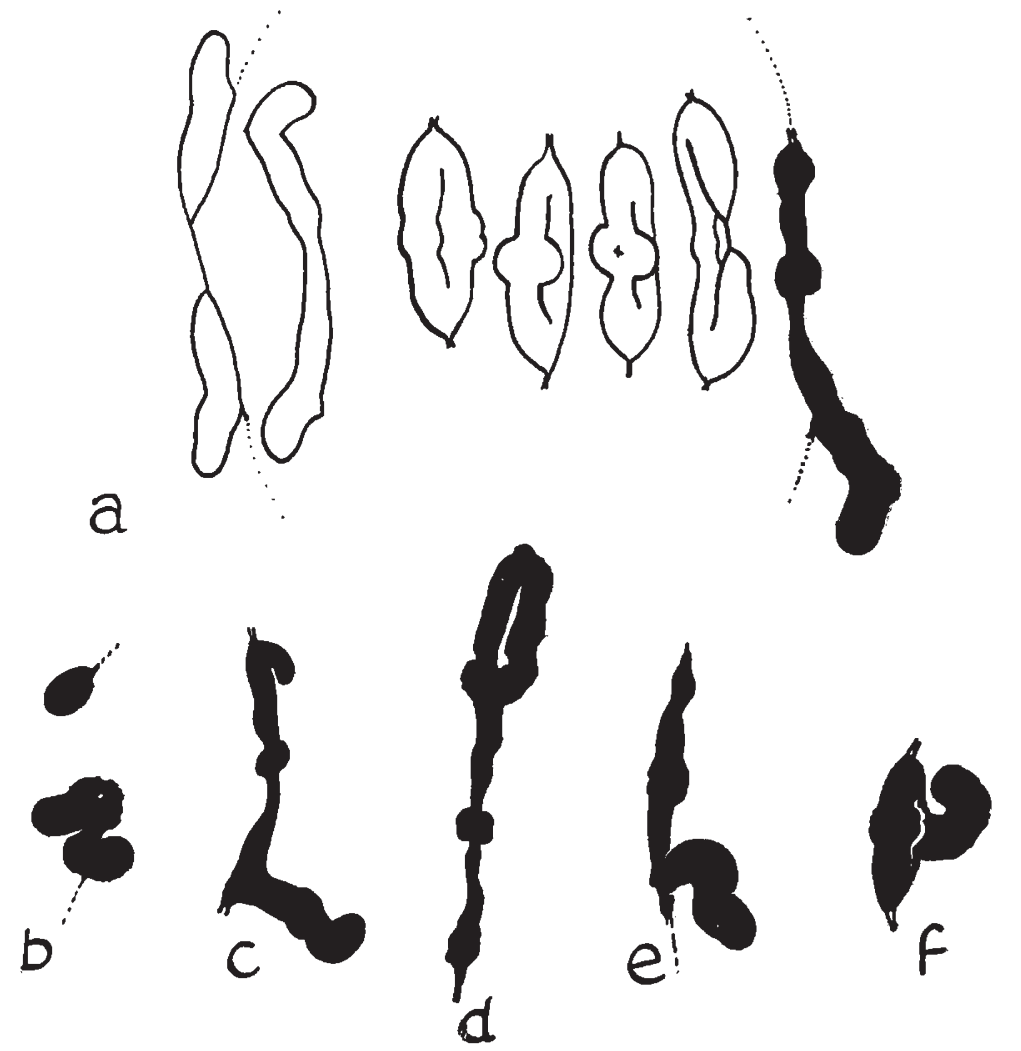

Fig. 1.-a. MI with breakage adjacent to the centromere in one bivalent. Acentric held by distal chiasma. $b$-e. Bivalents with breaks near to the centromere. $f$. Intercalary break between two chiasmata. $\times 2100$.

As a result of this distortion, bivalents become asymmetrical (see fig. I). They are similar to those produced by X-raying pachytene chromosomes in Uvularia (Darlington and La Cour, 1953); and in Uvularia, as in rye, the broken chromosomes release few, if any, fragments before first anaphase (see also Haque, 1953). The majority of breaks become detectable only at anaphase, when the fragments are released and bridges appear. The broken pieces until then are held, undetectable, in the bivalents. Rare associations of three or four chromosomes in p.m.c. of three plants in $\mathrm{Pr}_{3}$ can be interpreted either as $\mathrm{B}^{\prime \prime}$ or $\mathrm{B}^{\prime}$ (chromatid) breaks followed by interchange.

Table I gives the distribution of free fragments at first and second anaphases in p.m.c. of two plants. Occasional fragments remain attached by chiasmata at first anaphase (fig. $4 c$ ) and this probably 
is why the relative frequencies of fragments at second anaphase are greater than at first anaphase in the two plants. In table 2 are the frequencies of fragments with and without bridges. Bridges without

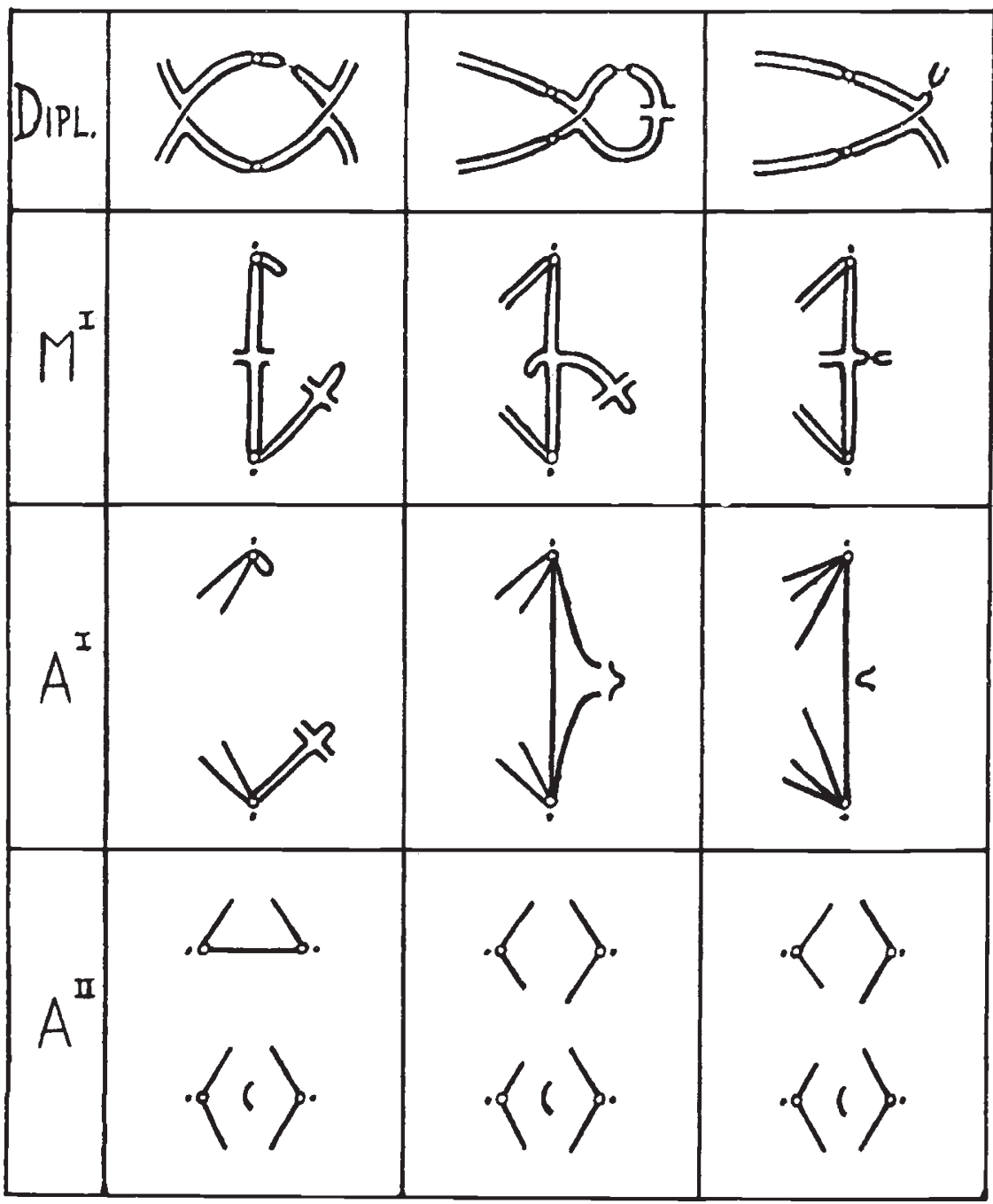

Fig. 2.-Diagram showing the consequences of $B^{\prime \prime}$ breakage with sister reunion at prophase. Breaks between the centromere and a chiasma, or between two chiasmata are manifest at first metaphase. Others show up only as bridges and fragments at anaphase. First division and second division bridges follow equational and reductional separation respectively at the point of breakage.

fragments, resulting from splitting errors, are not included in the table. They comprise about 5 per cent. of the total bridges at first anaphase.

The sequence of events, breakage and sister reunion, postulated to account for these observations is represented in fig. 2. Reunion in centrics gives rise to the anaphase bridges, which will appear with 
the fragments at the first anaphase when the distribution of chiasmata in the broken bivalents is such as to make separation at the breaks equational. Elsewhere, with reductional first division separation, the bridges show up at the second anaphase.

TABLE I

The frequency distributions of fragments in p.m.c. at $A I$ and $A I I$ in two plants

\begin{tabular}{|c|c|c|c|c|c|c|c|c|c|c|}
\hline \multirow{3}{*}{ Plant } & \multicolumn{5}{|c|}{ No. of AI fragments } & \multicolumn{4}{|c|}{ No. of AII fragments } \\
\cline { 2 - 7 } & 0 & 1 & 2 & 3 & 4 & 0 & 1 & 2 & 3 & 4 \\
\hline$P_{1} 3^{1}$ & 47 & 43 & 5 & 1 & 0 & 39 & 22 & 9 & 1 & 1 \\
$P_{1} 3^{2}$ & 44 & 14 & 5 & 0 & 0 & 35 & 18 & 2 & 2 & 0 \\
\hline
\end{tabular}

Under these conditions, it is clear that a chiasma proximal to a break with sister reunion cannot terminalise. Terminalisation will be limited by the formation of lateral chiasmata (figs. $3,4^{a}, b$ ). Since the majority of breaks as we shall see are near to the ends of the chromosomes, we would expect the average terminalisation value to be reduced. When we compare $\mathrm{P}_{13}$ with $\mathrm{P}_{14}$, which is relatively
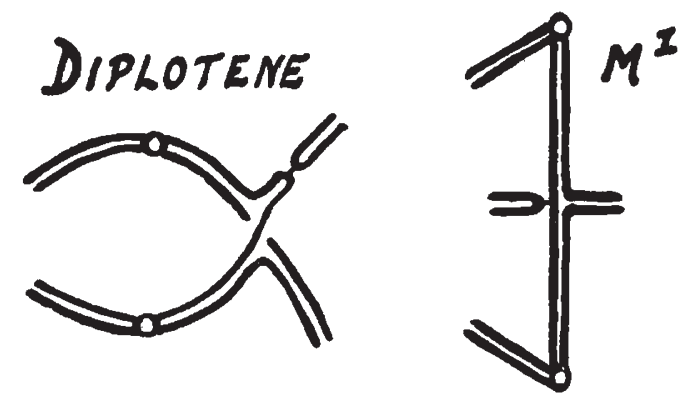

Fig. 3.-Diagram showing the formation of a lateral chiasma, and the arrest of terminalisation following prophase breakage and reunion.

normal, we do in fact find a significantly lower terminalisation value accompanying the breakage in $\mathrm{P}_{13}$. The terminalisation was scored for this comparison as the proportion of terminal chiasmata to the number of bivalent arms paired. The mean values are 0.62 for five plants in $\mathrm{P}_{1} 3$ and 0.78 for five plants in $\mathrm{P}_{14}$. After transforming these proportions to angles, using Fisher and Yates' table, a test of significance can be made, and this gives a probability of less than 0.001 .

Breakage in $\mathrm{P}_{1} 3$ is not accompanied by any alteration in chiasma frequency, the values for $\mathrm{P}_{1} 3$ and $\mathrm{P}_{14}$ showing no significant difference. In other cases of natural and induced meiotic breakage a change in chiasma frequency is often associated with breakage (see Darlington and La Cour, 1953). Abnormal proximal chiasmata in some P13 bivalents (see Rees, r955) may be secondary consequences of the 
disturbance causing breakage, though occasional chiasmata are proximal in $\mathrm{Pr}_{4}$, and in other lines without breakage.

This interpretation of the structural change assumes that all anaphase as well as metaphase configurations are consequences of single $\mathrm{B}^{\prime \prime}$ breaks. Certainly, only such $\mathrm{B}^{\prime \prime}$ breaks could account for the asymmetrical metaphase bivalents. It is, however, conceivable that some of the anaphase configurations might arise from other causes. If they do, the evidence indicates that these alternative causes, at most, account for only a small proportion of the fragments and bridges at anaphase. These alternatives are :-

(I) Pairing is between non-homologous chromosomes, i.e. intrahaploid (see Darlington, I937). Intra-haploid pairing is extremely unlikely in $\mathrm{PI}_{3} 3$ in view of the relatively high chiasma frequency (about $\mathrm{I} \cdot 8$ per bivalent) indicating efficient pairing.

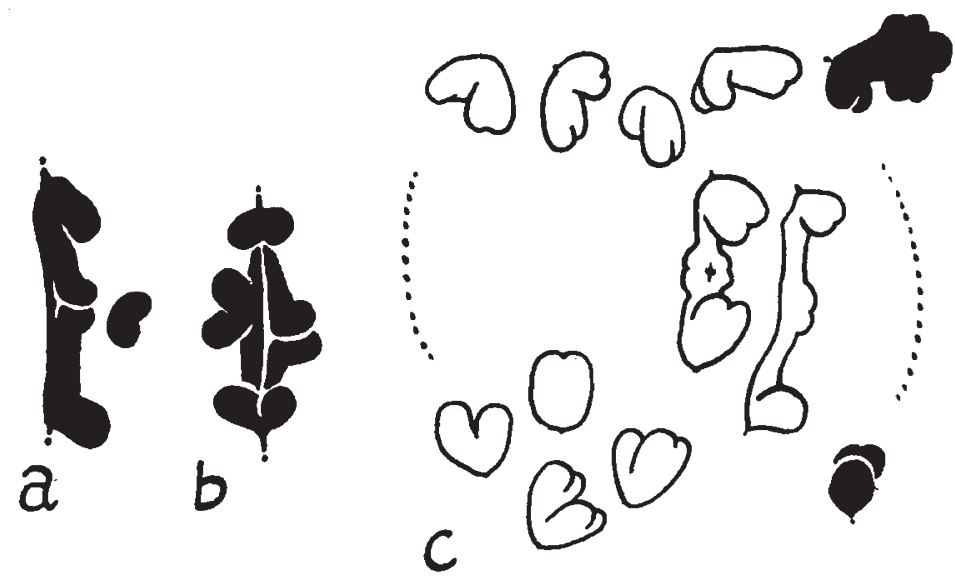

Fic. 4.- $-a, b$. Bivalents at early anaphase each with a lateral chiasma, a bridge and a fragment. The fragment has been released in $a$, but not in $b$. $c$. AI following $\mathrm{B}^{\prime \prime}$ breakage and reunion between the centromere and a chiasma in one bivalent. The acentric is held by the chiasma. $\times 2100$.

(2) PI3 plants are heterozygous for a number of inversions. A high degree of inversion heterozygosity after 29 generations of inbreeding would be remarkable, and it is ruled out on the following grounds. First p.m.c. of all twenty $\mathrm{F}_{1}$ plants between $\mathrm{P}_{13}$ and three other lines are normal at anaphase : bridges and fragments appear in less than I per cent. of cells. Secondly, since all PI3 plants have many fragments and bridges in p.m.c. this would imply that all $\mathrm{P}_{\mathrm{I}} 3$ plants were structural heterozygotes, and consequently that the structural homozygotes were lethal. But the closely related PI4 plants have no, or rare, anaphase bridges and fragments. They are not lethal and yet they are more or less completely homozygous with regard to chromosome structural arrangements.*

* In Chorthippus (Darlington, 1936) anaphase bridges and fragments were attributed to inversion crossing over. Darlington, however, pointed out that not all cells examined at pachytene showed inversion pairing. Some of the fragments and bridges therefore may well have resulted from the kind of $B^{\prime \prime}$ breakage described in this paper. 
(3) Multiple B" breaks occur before pachytene producing numerous inversions, followed by normal crossing over to give rise to bridges and fragments. This is also unlikely in view of the normality of the progeny on outcrossing $\operatorname{P}_{1} 3$.

(4) Normally paired chromatids break and reunite in the form of chiasmata with inverted crossing over (see Darlington and La Cour, 1953). We cannot entirely rule out inverted crossing over with normal pairing, but the following evidence shows it could at most be rare. In $\mathrm{P}_{13}$, where more than two chiasmata never form in one arm, an inverted cross over could give rise to a second division bridge only when a normal chiasma is proximal and disparate in relation to it. In the plant PI $3^{1}$, the proportion of arms with two and with one chiasma is $19: 227$ (in twenty p.m.c.) which corresponds to the

TABLE 2

The frequencies of fragments with and without bridges at $A I$ and $A I I$

\begin{tabular}{|c|c|c|c|c|c|c|}
\hline \multirow{2}{*}{ Plant } & \multicolumn{3}{|c|}{ AI } & \multicolumn{3}{|c|}{ AII } \\
\cline { 2 - 6 } & $\mathrm{br}+\mathrm{f}$ & $\mathrm{f}$ & $\mathrm{cells}$ & $\mathrm{br}+\mathrm{f}$ & $\mathrm{f}$ & whole p.m.c. \\
\hline $\mathrm{P}_{13^{\mathrm{i}}}$ & $\mathrm{II}$ & 5 & 28 & $\mathrm{I} 3$ & 34 & 72 \\
$\mathrm{P}_{13^{2}}$ & 17 & 7 & 77 & 4 & 24 & 66 \\
\hline
\end{tabular}

maximum proportion one would expect of fragments with bridges at second division to those without bridges. The observed proportion is significantly larger, $I_{3}: 34(\mathrm{P}=<0.00 \mathrm{I})$. Similarly in $\mathrm{P}_{1} 3^{2}$ the maximum expected is $5: 239$, and the observed $4: 24(\mathrm{P}=<0 \cdot 00 \mathrm{I})$. The relative excess of second anaphase bridges is also good evidence against (2) and (3). Upcott used the same type of analysis in 1937.

We are clearly justified in the assumption that predominantly, if not entirely, $\mathbf{B}^{\prime \prime}$ breakage of the kind represented in fig. 2 accounts for the abnormalities at all stages. We can thus confidently extend the analysis to a more detailed consideration of the observations.

\section{REUNION}

Acentrics.-At first anaphase in $\mathrm{P}_{\mathrm{I}} 3$ more than 95 per cent. of the fragments do not divide, so that SR occurs in at least 95 per cent. of acentrics.

Centrics.-In centrics the degree of SR was estimated by comparing the proportions of fragments and of bridges per p.m.c. (obtained from table 2). The proportions of fragments, in view of the attachment by chiasmata and consequent uncertainty of scoring of some acentrics at first anaphase, were calculated from second anaphase data.

In centrics bridges from SR can show either at the first or at the second anaphase. To estimate SR therefore the proportions of 
bridges at each anaphase are summed. The total in $\mathrm{P}_{1} 3^{1}$ is 0.57 . The proportion of fragments is 0.65 . We see that bridges per p.m.c. are almost as frequent as fragments. Clearly SR in centrics, like acentrics, approaches completeness. In $\mathrm{P}_{1} 3^{2}$, the proportion of bridges is 0.28 and fragments $0.4^{2}$. SR, though less than in $\mathrm{P}_{1} 3^{1}$, is still of a high order (about 70 per cent.). These values most probably underestimate SR in centrics, because short bridges break at early anaphase and are liable to be unrecorded.

This high proportion of bridges to fragments also indicates that if single chromatid breaks occur to produce fragments, they must be comparatively few. Otherwise, fragments would greatly exceed bridges, for with single $\mathbf{B}^{\prime}$ breaks there are no anaphase bridges.

In $\mathrm{P}_{1} 3^{1}$ and $\mathrm{P}_{1} 3^{2}$ about 70 per cent. of the fragments at first anaphase are accompanied by a bridge (table 2). With $B^{\prime \prime}$ breaks and SR this implies equational separation at the break in about

TABLE 3

The length distribution of acentrics measured at AII (30 cells)

\begin{tabular}{|c|c|c|c|c|c|c|c|c|c|c|c|c|}
\hline Length in $\mu$ & . & o & - & $I \cdot 5$ & - & $3 \cdot 0$ & - & 4.5 & $-6 \cdot 0$ & -7.5 & -9.0 & $-\quad \mathrm{II} \cdot 5$ \\
\hline Fragments & . & & 19 & & 21 & & 2 & & I & o & o & I \\
\hline
\end{tabular}

70 per cent. of cases. Since most breaks as will be shown are near the chromosome ends, this order of separation is exactly as one would expect with a chiasma frequency of between o and 2 per arm in $\mathrm{P}_{13}$, with a mean of just less than $\mathrm{I}$.

\section{THE DISTRIBUTION OF BREAKS}

With breakage at random, equal numbers of breaks would be expected in all regions of the chromosomes. The actual distribution of breaks, which can be deduced from fragment size (table 3 ), differs significantly from this expectation $(P=<0 \cdot 00 \mathrm{I})$. Small fragments are in excess. The average arm length of rye chromosomes is $4.5 \mu$ at second anaphase. With $B^{\prime \prime}$ breakage at random and SR, which approaches completeness, the average length of acentrics expected at second anaphase is about $4,5 \mu$. The value observed is less than $2 \mu$. Breakage, it appears, is localised towards the ends of the chromosomes. Alternatively, if the breakage is at random, restitution must be favoured proximally. Either way there is no doubt of spatial differentiation.*

While the anaphase data establish a pronounced distal localisation, it would appear from two sets of metaphase observations that a region

* In Tulipa (Darlington and Upcott, 194I) an excess of " minute" fragments produced by spontaneous breakage was attributed to intercalary double breaks and not to distal localisation. In Tulipa, however, the circumstances were different; (I) breakage per nucleus was high, and (2) "minute" acentrics showed an absence of SR. Neither condition holds for rye and therefore the small average size of the fragments must reflect distal localisation. 
of secondary localisation lies immediately adjacent to the centromeres. Thus, in one plant, 7 out of 12 asymmetrical bivalents (in 146 p.m.c.) have broken at this point, and 5 out of 6 breaks (in I 34 p.m.c.) in another plant (figs. I $a, b, d, e$; plate fig. 2).

There is evidence that prophase damage induced by $\mathrm{X}$-rays is localised in certain cases (La Cour, I953; Rees, I953), but time of breakage is not limited for non-spontaneous localised breakage : some chemicals and irradiations cause localised breaks in the resting stage (see Koller, 1954).

A natural breakage described by Haga (1953) in Paris is in many ways similar to that in rye. The breakage, predominantly $\mathrm{B}^{\prime \prime}$ with a high degree of SR, produces asymmetrical bivalents at metaphase and bridges and fragments at anaphase.

In rye and Paris the distribution of breakage, mainly distal and proximal respectively, corresponds in general with the localisation of chiasmata in the two species. It would appear therefore that similar factors may influence both the breakage and chiasma formation. Indeed it may well be that the distribution of breaks, as well as of chiasmata (see Darlington, 1935), corresponds with the distribution of torsion at pachytene.

\section{INHERITANCE AND GENE ACTION}

Each of fifteen $\mathrm{P}_{1} 3$ plants examined over the past three generations exhibits breakage : evidently the sub-line breeds true. On the simplest view the divergence from $\mathrm{P}_{4} 4$ could be interpreted as a single gene mutation. On crossing $P_{1} 3$ with other lines the $F_{1} s$ are normal at anaphase, with rare or no bridges and fragments. At metaphase asymmetrical bivalents were found in rare p.m.c. in four out of twenty $F_{1}$ plants. In an $F_{2}$ raised from one cross only one out of twenty-two plants showed evidence of breakage other than by bridges and fragments in exceptional p.m.c. (an average of less than one per cent.). Consequently, if a single gene mutation is primarily responsible for the divergence, then its effect must be highly sensitive to its genetic background in view of its low penetrance in $\mathrm{F}_{2}$. There is, therefore, no doubt that the abnormality is influenced by at least two genes, probably many.

The localisation of the breakage, whether we attribute it to (I) mechanical or physical differences along the chromosomes, which are becoming increasingly well known (e.g. Lima de Faria, 1952), or (2) chemical differences in the chromosome matter, reflects the specificity of gene effect within the nucleus, and emphasises the phenotypic aspect of chromosome differentiation.

\section{SUMMARY}

I. A type of chromosome breakage in inbred rye (line $\mathrm{PI}_{3}$ ) is genetically determined. Breakage is interpreted as $\mathrm{B}^{\prime \prime}$ (chromosome), probably during pachytene. SR (sister reunion) in acentrics 
I, 2. MI's each with an asymmetrical bivalent following breakage between the centromere and a chiasma. $\times 2100$.

3. Sister AII's with a short bridge (left) and a long fragment (right) as a result of breakage with reunion near to the contromere, with reductional separation at the break at AI. $\times$ ir6o. 


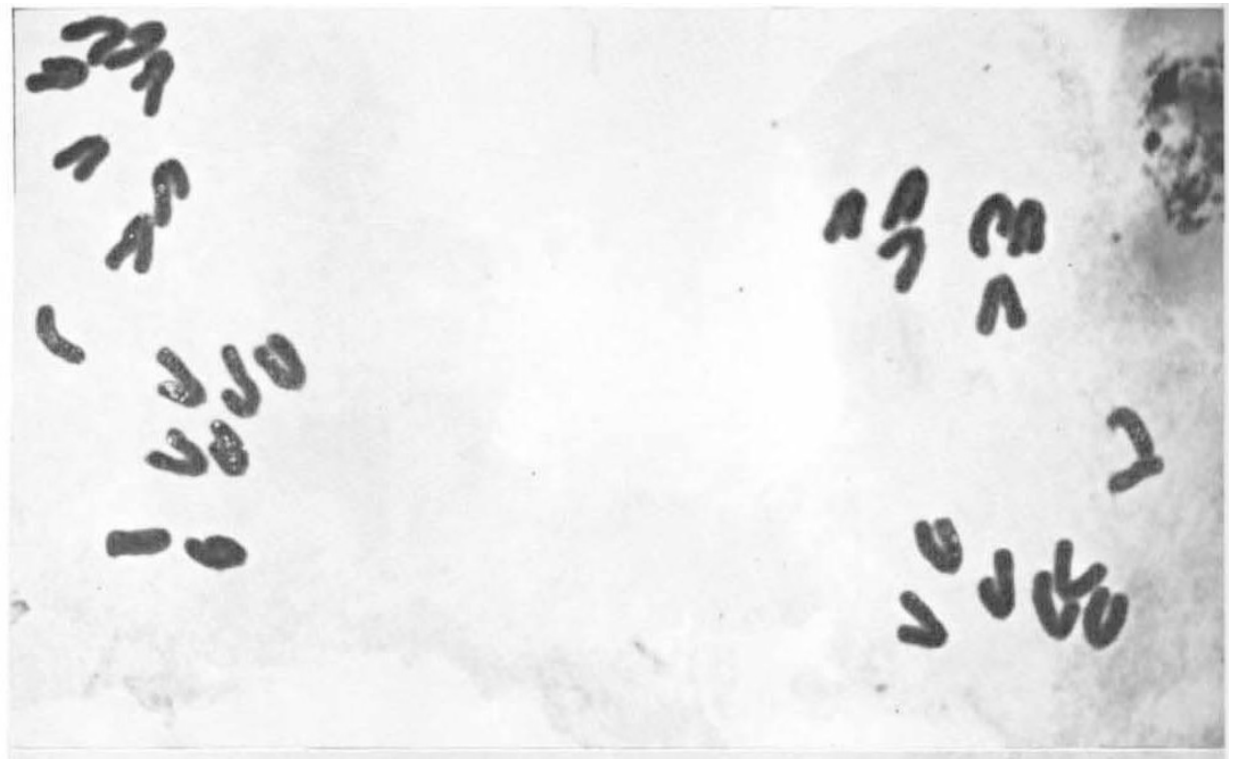

,
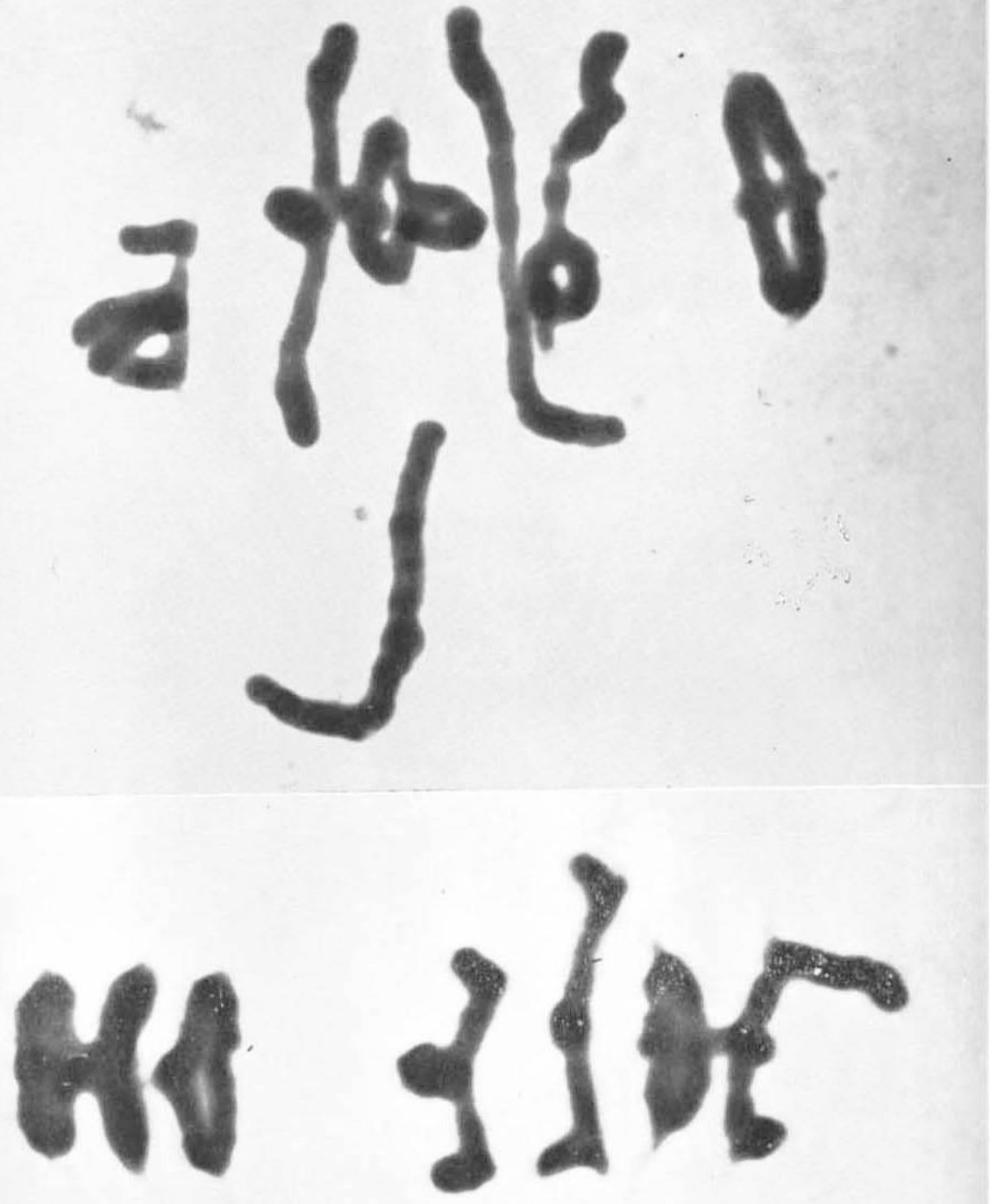
approaches completeness. In centrics $\mathrm{SR}$ is approximately 70 per cent. in one plant and almost complete in another.

2. About 5 per cent. of the breaks are visible in metaphase bivalents, when the broken ends are forced apart. This happens when the break is (i) between the centromere and a chiasma, or (ii) between two chiasmata.

3. The 95 per cent. of breaks distal to chiasmata show up at anaphase when the acentrics are released and the centrics form bridges.

4. After breakage and reunion in distal segments of the bivalents terminalisation of chiasmata at first metaphase is limited by the formation of lateral chiasmata.

5. Crossing experiments indicate that more than one gene controls the abnormality.

6. Breakage is localised mainly away from the centromeres towards the ends of the chromosomes, agreeing closely with the distribution of chiasmata.

Acknowledgments.-We are indebted to Professor C. D. Darlington and Professor K. Mather for helpful criticism.

\section{REFERENCES}

DARLIngton, G. D. 1935. The internal mechanics of the chromosomes. P.R.S., $\mathrm{B}, I I 8,33-96$.

DARLINGTON, C. D. 1936. Crossing-over and its mechanical relationships in Chorthippus and Stauroderus. 7. Genet., 33, 465-50o.

Darlington, C. D. 1937. Recent Advances in Cytology. 2nd ed. London: J. \& A. Churchill.

DARLINGTON, C. D., AND UPGOTT, M. B. I941. Spontaneous chromosome change. 7. Genet., 4I, 297-338.

DARLINGTON, C. D., AND LA COUR, L. F. 1953. The classification of radiation effects at meiosis. Heredity, 6, Suppl., $4^{1-55}$.

HAGA, T. 1953. Meiosis in Paris. II. Spontaneous breakage and fusion of chromosomes. Cytologia, $18,50-66$.

HaQue, A. 1953. The irradiation of meiosis in Tradescantia. Heredity, 6, Suppl., 57-75.

Koller, P. G. 1954. Chromosome breakage. Progress of Biophysics and Biophysical Chemistry, 4, 195-243.

LA COUR, L. F. I 953 . Chromosome breakage experiments with endosperm : subchromatid breakage. Nature, 172,501 .

LAMM, R. 1936. Cytological studies on inbred rye. Hereditas, 3I, 21 7-240.

LIMA DE FARIA, A. 1952. Chromomere analysis of the chromosome complement of rye. Chromosoma, 5, I-68.

MÜNTZING, A., AND AKDIK, s. I 948 . Cytological disturbances in the first inbred generation of rye. Hereditas, 34, 485-509.

REES, H. 1953. Centromere control of chromosome splitting and breakage. Heredity, 6, Suppl., 235-245.

REES, H. 1955. Genotypic control of chromosome behaviour in rye. I. Inbred lines. Heredity, 9, 93-1 16.

upcotт, м. в. 1937. The genetic structure of Tulipa. II. Structural hybridity. 7. Genet., 34, 339-399.

WALTERS, S. M. 1951. Spontaneous chromosome breakage and atypical chromosome movement in meiosis of the hybrid Bromus marginatus $\times B$. pseudolaevipes. Genetics, 37, 8-25. 